\title{
Optimization of Sandwich Composites Fuselages Under Flight Loads
}

\author{
Chongxin Yuan • Otto Bergsma • Sotiris Koussios • \\ Lei $\mathrm{Zu} \cdot$ Adriaan Beukers
}

Received: 19 September 2010 / Accepted: 13 October 2010 / Published online: 24 November 2010

(C) The Author(s) 2010. This article is published with open access at Springerlink.com.

\begin{abstract}
The sandwich composites fuselages appear to be a promising choice for the future aircrafts because of their structural efficiency and functional integration advantages. However, the design of sandwich composites is more complex than other structures because of many involved variables. In this paper, the fuselage is designed as a sandwich composites cylinder, and its structural optimization using the finite element method (FEM) is outlined to obtain the minimum weight. The constraints include structural stability and the composites failure criteria. In order to get a verification baseline for the FEM analysis, the stability of sandwich structures is studied and the optimal design is performed based on the analytical formulae. Then, the predicted buckling loads and the optimization results obtained from a FEM model are compared with that from the analytical formulas, and a good agreement is achieved. A detailed parametric optimal design for the sandwich composites cylinder is conducted. The optimization method used here includes two steps: the minimization of the layer thickness followed by tailoring of the fiber orientation. The factors comprise layer number, fiber orientation, core thickness, frame dimension and spacing. Results show that the two-step optimization is an effective method for the sandwich composites and the foam sandwich cylinder with core thickness of $5 \mathrm{~mm}$ and frame pitch of $0.5 \mathrm{~m}$ exhibits the minimum weight.
\end{abstract}

Keywords Sandwich $\cdot$ Composites $\cdot$ Stability $\cdot$ Optimization $\cdot$ ANOVA

\section{Nomenclature}

$t_{\mathrm{f}} \quad$ The skin thickness

$t_{\mathrm{c}} \quad$ The core thickness

$h \quad$ Sandwich thickness $h=t_{\mathrm{c}}+t_{\mathrm{f}}$

$E_{\mathrm{f}} \quad$ Normal modulus of the faces

$E_{\mathrm{c}} \quad$ Normal modulus of the core

$G_{\mathrm{f}} \quad$ Shear modulus of the faces

$G_{\mathrm{c}} \quad$ Shear modulus of the core

$\nu_{\mathrm{f}} \quad$ Poison ratio of the faces

$\nu_{\mathrm{c}} \quad$ Poison ratio of the core

C. Yuan $(\bowtie) \cdot$ O. Bergsma $\cdot$ S. Koussios $\cdot$ L. Zu $\cdot$ A. Beukers

Section Composites, Faculty of Aerospace Engineering,

Delft University of Technology, Delft, The Netherlands

e-mail: yuanchongxin@yahoo.com.cn 
$t_{\text {eq }} \quad$ Equivalent thickness $t_{e q}=\sqrt[3]{6 t_{f} h}$

$I_{\mathrm{t}} \quad$ Moment of inertia $I_{\mathrm{t}}=t_{\mathrm{f}} \mathrm{h}^{2} / 2$

$I_{\mathrm{f}} \quad$ Moment of inertia of the face $I_{\mathrm{f}}=t_{\mathrm{f}}^{3} / 6$

$\lambda \quad \lambda=1-v_{\mathrm{f}}^{2}$

\section{Introduction}

Due to their high structure efficiency, sandwich structures have been increasingly applied in aerospace engineering. In 1924, Karman and Stock were granted a patent for a glider plane having a sandwich fuselage. The Beech Starship used Nomex honeycomb throughout its structures. The famous aircraft Voyager, which flew around the world in 9 days without refueling, was basically made from honeycomb and carbon tape prepreg for each skin[1]. Moreover, the sandwich structures were also used as helicopter blades and fuselage. Nevertheless, they were still not widely used in large civil airplane because of design and manufacture uncertainties. However, sandwich construction combined with composite materials will be a novel concept for the future fuselage structures. Van Tooren[2] proved the feasibility of sandwich structures to be used as fuselages of large commercial airplanes. The future airplane should be safer, quieter, greener and more cost, -effective, thus demands for lightweight and functional structure would be stronger. The sandwich structure is a better choice because of its higher stiffness-to-weight ratio, superior fatigue strength, larger acoustic and thermal insulation[3]. In the Advanced Technology Composite Aircraft Structures (ATCAS) program, the sandwich structure was adopted to produce the keel and sides panels considering its weight and cost advantage toward the stringer-stiffened skin[4].

The mechanical analysis of sandwich structures can be divided into two major parts, one is dealing with stability problems and the other is with the resulting stresses and strains in the faces and core. NASA[5] issued a manual on the structural stability analysis of sandwich structures under axial compression, pure bending, external pressure, torsion and transverse shear, and published a report concerning the buckling of thin-walled circular cylinders[6]. The buckling of thin-walled isotopic cylinders under combined torsion and pressure were studied by ESDU[7]. The interactions between torsion and pressure were analyzed and the results showed that increasing inner pressure of cylinder raised the torsion buckling stress. Lennon[8] investigated the effect of axial compression and pressure on torsion buckling behavior. The compression buckling for axisymmetric imperfect sandwich cylinders was studied by Tennyson[9], the equilibrium and compatibility equations were built as two governing equations in terms of $w$ (displacement) and $F$ (applied load). Peterson and Anderson[10] carried out three buckling tests for the honeycomb sandwich structures under bending. However, few studies have so far focused on the stability and optimization of the sandwich cylinder with composites facings.

In this paper, the mathematical formulae are built to predict the stability of sandwich cylinder under pure bending, torsion, and transverse shear, respectively. Based on the typical dimensions and load cases of A320, the sandwich cylinders are optimized with iterative design procedure. Next, results for stability predicted by a FEM model are compared to that by the mathematical formulae. Based on the FEM model, a two-step optimization for the sandwich composites cylinders is conducted. The results show that too many layers of the composites are needed to satisfy the buckling constraints. The frames are thus added to the cylinder shell to investigate whether they can improve the structural efficiency. Subsequently, by the comparison among different core materials the Rohacell $200 \mathrm{WF}$ foam is chosen for the sandwich core. Finally an analysis of variance (ANOVA) design between frame spacing and core thickness is presented. 


\section{Analytical Formulae and Optimization}

\subsection{Theoretical Formulation}

In this study, the sandwich composites structure is assumed to exhibit a linear stress-strain relationship. Once the applied load is removed, the structure will return to its original position. However, if the loads exceed their threshold, the structure may become unstable. In this case, the structure continues to deflect without increasing of the magnitude of the applied loads, because the structure has actually buckled. Hence, the term "instability" is also called "buckling". In the following section, the theoretical buckling prediction of the sandwich cylinders under different loading cases and the interaction for combined loads will be discussed.

\subsection{Facesheet Wrinkling}

Facesheet wrinkling is usually viewed as a local, short wavelength buckling phenomenon. The facesheet can deform symmetrically or anti-symmetrically. Most of theoretical analyses for the facesheet wrinkling are based on the mathematical model of uniaxially loaded flat sandwich strut. For sandwich structures under combined loads, the procedure of the prediction for the onset of wrinkling is to calculate the maximum principle compressive stress of the facesheet. Lopatin and Morozov[11] present the solution of symmetrical face sheet wrinkling for sandwich panels with composite facings and an orthotropic core. The common expression of theoretical wrinkling stresses for sandwich struts with thick cores is given by:

$$
\sigma_{w r}=k\left(E_{f} E_{c} G_{c}\right)^{1 / 3}
$$

where $k$ varies from 0 to 1 according to different theories and boundary conditions. Ley[12] gave a comprehensive summary for different mathematical models. Here the $k$ is 0.5 .

\subsection{Bending}

Bending buckling is triggered by the compressive component of bending moment. For the cylinder, the interaction between shear crimping and compressive buckling has been taken into account. Stein and Mayers[13] developed the following equations for the critical buckling stress under compression:

Flexural stiffness:

$$
D_{S}=\frac{E_{f} t_{f} h^{2}}{2\left(1-v_{f}^{2}\right)}
$$

Transverse shear stiffness:

$$
D_{Q}=\frac{G_{c} h^{2}}{h-t_{f}}
$$

Curvature parameters:

$$
\begin{gathered}
z_{a}=\sqrt{\frac{2 t_{f} L^{4}\left(1-v_{f}^{2}\right)}{R^{2} I_{S}}} \\
r_{a}=\frac{D_{S} \pi^{2}}{D_{Q} L^{2}}
\end{gathered}
$$


Compressive load coefficients:

$$
k_{x a}=\left\{\begin{array}{cc}
\frac{1}{1+r_{a}}+\frac{z_{a}^{2}}{\pi^{4}} & \frac{z_{a}}{\pi^{2}} \leq \frac{1}{1+r_{a}} \\
\frac{z_{a}}{\pi^{2}}\left(2-\frac{z_{a} r_{a}}{\pi^{2}}\right) & \frac{1}{1+r_{a}}<\frac{z_{a}}{\pi^{2}}<\frac{1}{r_{a}} \\
\frac{1}{r_{a}} & \frac{z_{a}}{\pi^{2}} \geq \frac{1}{r_{a}}
\end{array}\right.
$$

Knockdown factor:

$$
\eta_{b}=1-0.731\left(1-e^{\frac{-1}{16 \sqrt{R / t_{e q}}}}\right)
$$

Critical compressive stress resultant:

$$
N_{x}=k_{x a} \frac{D_{S} \pi^{2}}{L^{2}} \eta_{b}
$$

Bending moment:

$$
M=2 \pi R^{2} N_{x}
$$

where $\eta_{\mathrm{b}}$ is the knockdown factor for initial imperfections.

\subsection{Torsion}

March and Kuenzi[14] developed an energy method to determine the shear buckling stress under torsion. The small-deflection theory is used to find the solution of the energy equilibrium equation. For a sandwich cylinder with significantly thin skins, the critical shear stress can be evaluated by the following formula:

$$
\tau_{c r}=K_{f} \eta E_{f} \frac{h}{R}
$$

For isotropic facings and cores, $K_{\mathrm{f}}$ can be given by

$$
K_{f}=\psi_{1}+\psi_{2}
$$

in which

$$
\begin{gathered}
\psi_{1}=\frac{J \rho^{2}}{4 \pi^{2} x}\left[\frac{(x-\rho)^{4}}{\left[1+(x-\rho)^{2}\right]^{2}}+\frac{(x+\rho)^{4}}{\left[1+(x+\rho)^{2}\right]^{2}}\right] \\
\psi_{2}=\frac{\pi^{2}}{\left(1-\frac{t_{c}}{h}\right) 4 J \rho^{2} x}\left[\frac{\frac{I_{t}}{h^{3}}\left[A_{1}+2 A_{2}+A_{3}+\left(A_{1} A_{3}-A_{2}^{2}\right) \frac{\pi^{2} \lambda}{J \rho^{2}}\left(\frac{S}{A_{4}}+S\right)\right]}{1+\frac{\pi^{2} \lambda}{J \rho^{2}}\left(S \frac{A_{1}}{A_{4}}+S A_{3}\right)+\left(\frac{\pi^{2} \lambda}{J \rho^{2}}\right)^{2} S^{2} \frac{A_{1} A_{3}-A_{2}^{2}}{A_{4}}}+\frac{I_{f}}{h^{3}}\left(A_{1}+2 A_{2}+A_{3}\right)\right] \\
J=\frac{L^{2}}{h R} \\
\phi=\frac{t_{c} t_{f}}{2} \\
\rho=\frac{\pi R}{n L}
\end{gathered}
$$




$$
\begin{gathered}
S=\frac{\phi E_{f}}{\lambda G_{c} R h} \\
A_{1}=\frac{2}{\lambda}\left(x^{4}+6 x^{2} \rho^{2}+\rho^{4}\right)+2 \frac{G_{f}}{E_{f}}\left(x^{2}+\rho^{2}\right) \\
A_{2}=2\left(\frac{v_{f}}{\lambda}+\frac{G_{f}}{E_{f}}\right)\left(x^{2}+\rho^{2}\right) \\
A_{3}=\frac{2}{\lambda}+2 \frac{G_{f}}{E_{f}}\left(x^{2}+\rho^{2}\right) \\
A_{4}=x^{2}+\rho^{2}
\end{gathered}
$$

here values of $x$ can be established for the minimization of $K_{\mathrm{f}}$. If the cylinder is very long, the value of $\rho$ approaches to zero, and the above equation can be simplified. And the torsion moment can be given by:

$$
T_{c r}=2 \pi R^{2}\left(t_{f}+t_{f}\right) \tau_{c r}
$$

\subsection{Shear}

In the case of isotropic cylinders, the lower-bound value for transverse shear load $\tau_{c r s}$ is 1.25 times of the critical torsion stress. Thereby, for a cylinder loaded with a transverse shear force the critical value can be computed by the following formula:

$$
\begin{gathered}
Q_{c r}=\pi R\left(t_{f}+t_{f}\right) \tau_{c r s} \\
\tau_{c r s}=1.25 \gamma_{s} K_{f} \eta E_{f} \frac{h}{R}
\end{gathered}
$$

where, $K_{\mathrm{f}}$ is the same as that for torsion.

\subsection{Buckling Interaction}

The interaction criterion among bending, torsion and transverse shear can be expressed by

$$
R_{c}=\frac{M}{M_{c r}}+\frac{T}{T_{c r}}+\frac{Q}{Q_{c r}}
$$

The buckling will occur when $R_{\mathrm{c}}>=1$

\subsection{Dimensioning Procedure of Sandwich Composites}

A program for dimensioning sandwich composites is carried out by the software Matlab. In the program, the core and skin thickness are varied to obtain the minimum total weight of 
the sandwich composites. The sizing procedures of sandwich cylinders are shown as in Fig. 1.

\section{FEM Analysis}

A cylinder model with length of $10 \mathrm{~m}$ and radius of $2 \mathrm{~m}$ is built to model the fuselage. The QUAD4 and HEX8 element are chosen to model the skin and core, respectively. Both the inner and outer skins consist of 500 QUAD4 elements and the core consists of 500 HEX8 elements. Since this study is not aimed at investigating the bonding strength between core and skins, it is assumed that the adhesive agent is sufficiently strong to prevent the delamination between core and skins. The core shares nodes with the two skins. The multipoint connector RBE3 is used to equally distribute the bending and torsion moments to the nodes around the circular end.

\subsection{FEM Approach for Linear Buckling}

In the finite element analysis, the overall structural stiffness is represented by:

$$
\begin{gathered}
{[K]=\left[K_{a}\right]+\left[K_{d}\right]} \\
{\left[K_{a}\right]=\sum_{i=1}^{N}\left[k_{a}\right]_{i}} \\
{\left[K_{d}\right]=\sum_{i=1}^{N}\left[k_{d}\right]_{i}}
\end{gathered}
$$

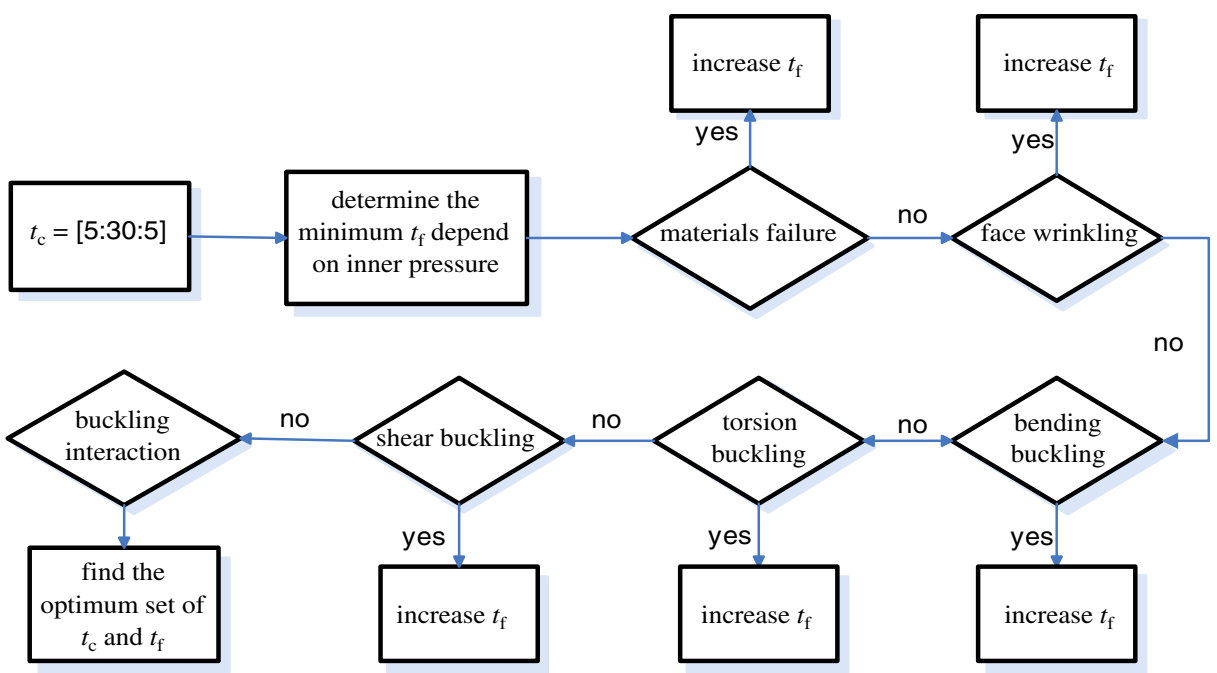

Fig. 1 Flow diagram of the sizing procedure for sandwich cylinders 
where $\left[K_{\mathrm{a}}\right]$ is the system linear stiffness matrix; $\left[K_{\mathrm{d}}\right]$ is the differential stiffness which denotes the higher-order terms of strain-displacement relationship; $\left[k_{a}\right]_{i}$ is the linear stiffness of the $\mathrm{i}$-th element; $\left[k_{d}\right]_{i}$ is the differential stiffness of the $i$-th element and it is a function of geometry, element type, and applied loads.

The total potential energy is given by:

$$
[U]=0.5\{u\}^{T}\left[K_{a}\right]\{u\}+0.5\{u\}^{T}\left[K_{d}\right]\{u\}
$$

where $\{u\}$ is the displacement vector. In order to achieve the static equilibrium of the structure, the potential energy has a stationary value. Therefore, the relationship can be formulated by:

$$
\frac{\partial[U]}{\partial u_{i}}=\left[K_{a}\right]\{u\}+\left[K_{d}\right]\{u\}=0
$$

where $U_{\mathrm{i}}$ is the displacement of the $\mathrm{i}$-th degree of freedom. The equation can then be rewritten as (detailed derivation can be referred to[15])

$$
\left|\left[K_{a}\right]+\lambda_{i}\left[K_{d}\right]\right|=[0]
$$

where $\|$ stands for the determination of the matrix, and the number of eigenvalue $\lambda_{i}$ is equal to the number of degree of freedom of the FEM model. The buckling load can be obtained by the equation:

$$
P_{c r_{i}}=\lambda_{i} P_{a}
$$

where $P_{\mathrm{a}}$ is the applied loads. In general, the lowest eigenvalue gains most interests, because it leads to the critical load and the structure fails before reaching higher buckling loads.

For certain load cases, the absolute value of the lowest eigenvalue should be larger than 1 to prevent buckling.

\subsection{Material Failure Criteria Applied in the Nastran}

In Nastran, the layer thickness, fiber orientation and stack sequence can be defined in the PCOMP entry. The stress, strain, failure index (including a bonding failure index) can be obtained. The Tsai-Wu failure criterion is here used to evaluate the composites failure.

$$
\left(\frac{1}{X_{t}}-\frac{1}{X_{c}}\right) \sigma_{1}+\left(\frac{1}{Y_{t}}-\frac{1}{Y_{c}}\right) \sigma_{2}+\frac{\sigma_{1}^{2}}{X_{t} X_{c}}+\frac{\sigma_{1}^{2}}{X_{t} X_{c}}+2 F_{12} \sigma_{1} \sigma_{2}+\frac{\sigma_{12}^{2}}{S^{2}}=1
$$

where, $X$ and $Y$ is the tensile/compressive strength in 1- and 2- directions, respectively. $S$ is the shear strength. Subscripts $t$ and $\mathrm{c}$ stand for the tension and compression respectively. $F_{12}$ should be determined experimentally unless it is provided. $\sigma$ is the actual stress.

In order to avoid the composites failure, the absolute value of left-hand side of Eq. 33 should be less than 1 .

\section{Verification of FEM Model}

In this section, the buckling loads obtained by analytical formulation and the FEM are compared to each other under the loading cases including axial compression, bending, shear and torsion. Subsequently, the optimization results of the FEM are compared to the results 
obtained by procedure in Section 2.6. In the FEM analysis, the material properties are exactly the same as in the analytical method and the face materials are quasi-isotropic. The material properties and load cases can be found in Appendix A.

\subsection{Comparison of Buckling Loads}

The length and radius of the cylinder are supposed to be $10 \mathrm{~m}$ and $2 \mathrm{~m}$ respectively, and the face and core thickness are $1 \mathrm{~mm}$ and $30 \mathrm{~mm}$, respectively. In the FEM, the mesh density is 25 by 20 and the boundary condition is simply supported. The results are shown in Table 1. It can be seen that for the bending and torsion loads, the buckling loads determined by the FEM show a reasonable agreement with those obtained from the analytical models. However, for the transverse shear force, a large difference can be observed.

\subsection{Comparison of Sizing Results for the Sandwich Cylinder}

The optimization of sandwich composites cylinders with FEM can be expressed as:

Minimize:

$$
M(x)
$$

Subject to:

$$
\begin{aligned}
& \operatorname{Abs}(\lambda)>1 \\
& \sigma(\mathrm{x}) / \mathrm{S}<1 \\
& x^{l}<x<x^{u}
\end{aligned}
$$

The objective function is the mass of the cylinder, and the constraints are the materials strength, local and global buckling. The variables are the skin thickness $t_{\mathrm{f}}$. The core thickness $t_{\mathrm{c}}$ increases from $5 \mathrm{~mm}$ to $30 \mathrm{~mm}$ by $5 \mathrm{~mm}$ interval.

In Fig. 2, the resulting $t_{\mathrm{f}}$ and weight are both displayed as $t_{\mathrm{c}}$ increases. It is revealed that the total sandwich structural weight is minimum when $t_{\mathrm{c}}=15 \mathrm{~mm}$. As $t_{\mathrm{c}}$ increases, $t_{\mathrm{f}}$ decreases with a nearly linear trend. The optimization results obtained by FEM and by analytical method show a good agreement. However, the current analytical and FEM optimization have such limitations:

1, the laminates are here assumed to be isotropic and thus the composite layup should be limited to obtain the quasi-isotropic property.

2, the buckling stability under internal pressure is not considered due to lack of proofing equations.

3, the criterion for the stability of the sandwich structure does not take the cylinder length into account.

Table 1 Buckling load computed by analytical and FEM

\begin{tabular}{llll}
\hline & Bending $(\mathrm{Nm})$ & Shear(N) & Torsion(Nm) \\
\hline analytical & $2.6 \mathrm{E} 7$ & $1.9 \mathrm{E} 6$ & $6.3 \mathrm{E} 6$ \\
FEM & $2.2 \mathrm{E} 7$ & $1.4 \mathrm{E} 6$ & $6.1 \mathrm{E} 6$ \\
Deviation & $16 \%$ & $26 \%$ & $3.2 \%$ \\
\hline
\end{tabular}


Fig. 2 Comparison of the optimization results between FEM and analytical method

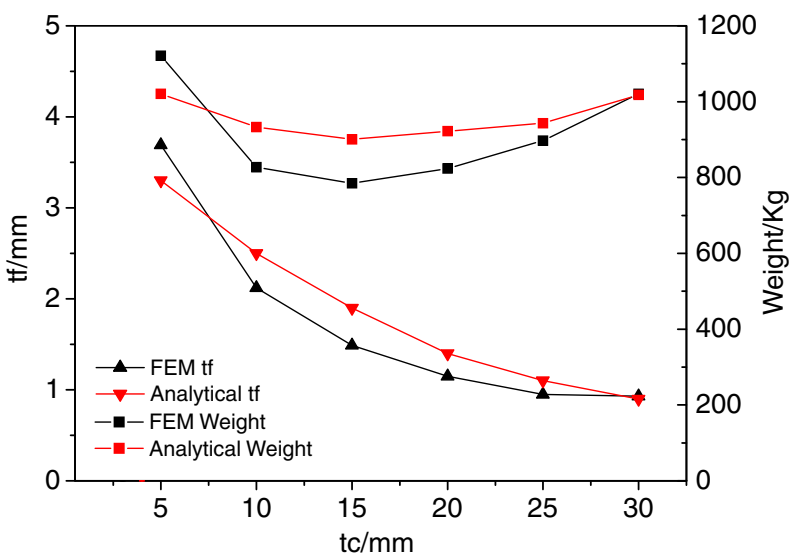

Therefore, the FE model need to be further developed to obtain a more accurate optimization model. In the following section, a more advanced model is built.

\subsection{Parametric Optimization of Sandwich Cylinder}

In this FEM analysis, both the fiber orientation and layer number will be taken as variables. Meanwhile, the internal pressure loads will be added. There are two steps: First, the fiber orientation is frozen and the layer thickness is optimized. The objective is the minimum total structural weight. The minimum laminates thickness (the layers number) can be determined by the first step. Secondly, the laminates thickness is frozen and the fiber orientation is optimized to attain a maximum buckling load. The FE model is shown in Fig. 3. In this model, the Tsai-Wu criterion is used to predict the composites failure. Because the materials in Appendix A do not supply the bonding shear stress limit $F_{12}$, another carbon/epoxy materials is here applied and its property is shown in Appendix B.

\subsubsection{Step 1: Determine the Minimum Layers Number}

At the beginning, the sandwich structure with $t_{\mathrm{c}} 20 \mathrm{~mm}$ is set as a baseline, and the initial layup is $[0 / 30 / 45 / 60 / 90]_{\mathrm{s}}$. As mentioned earlier, the laminate orientation is not changed but

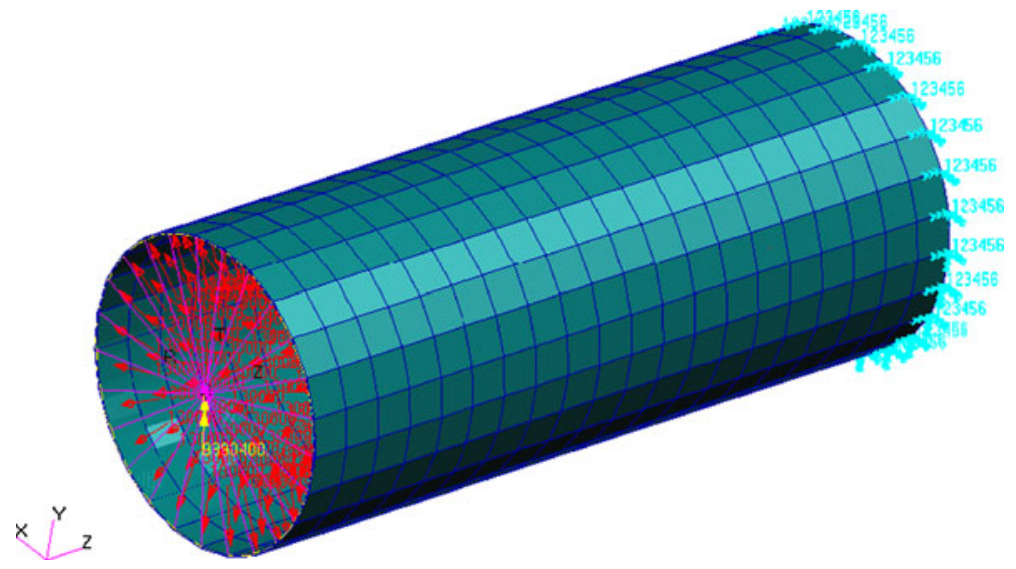

Fig. 3 The load cases and boundary condition of the FEM model 
the layer thickness is varied to satisfy the constraints of structural buckling and composites materials failure.

In the initial design, the maximum failure index is 2.03 (Fig. 4) and the buckling eigenvalue is -0.22 . To prevent the buckling of the structure, the absolute value of the buckling eigenvalue should be larger than 1 and the failure index is less than 1 . Therefore the maximum failure index decreases to be 0.506 (Fig. 5) and the buckling eigenvalue is -1.02 . After optimization for layer thickness, the initial layer number per skin is 10 and the final layer number is 34 .

Meanwhile, by comparison of the failure index of the Figs. 4 and 5, It is found find that the failure fringe is skew at the fixed end of the cylinder in Fig. 4, and the fringe is straighter in Fig. 5. This maybe is caused by the effect of the torsion moment as the material strain reduces when the thickness increase. It can also be found that the stress is not evenly distributed around the cylinder cross-section. The crown section undergoes the largest tension strain and its failure index is the largest. The side panel mainly endures the shear strain and its failure index is the lowest.

\subsubsection{Step 2: Determination of the Optimum Fiber Orientation}

As known from the first step, each skin consists of at least 34 layers in order to satisfy the buckling and composites failure criteria. In this section, the 34 layered laminate is built in the Patran. The layer orientations are chosen as the variables. The possible values of the orientations are $[0, \pm 30, \pm 45, \pm 60,90]$. Because the laminate is symmetric, only 17 variables are studied.

From Fig. 6, it can be shown that $0^{\circ}$ layup is the most effective for improving the buckling load, because bending moment is the preceding load compared with torsion moment. Under the bending moment, the sandwich cylinder mainly endures axial compression. And the elastic modulus of the $0^{\circ}$ layup is the largest in axial direction. However, it should be noted that as the number of the $0^{\circ}$ layup increases, the failure index rises. The increase of layup in the longitudinal direction enhances the stability of sandwich composites cylinder (Fig. 7).

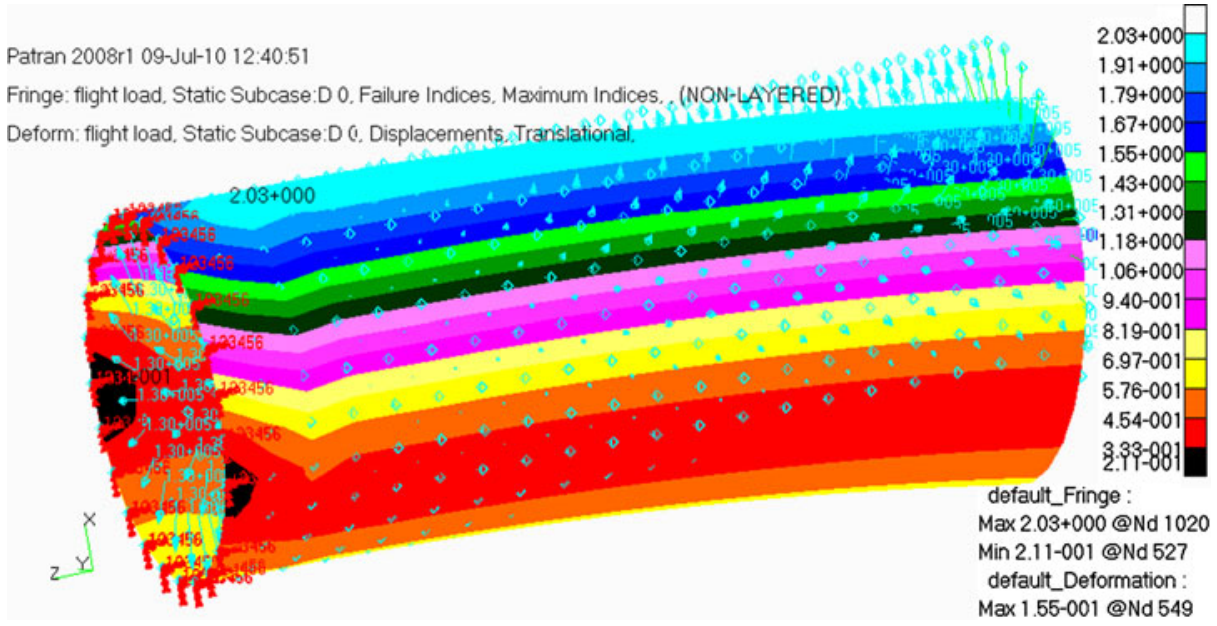

Fig. 4 The deformation and maximum failure indices at the initial design 


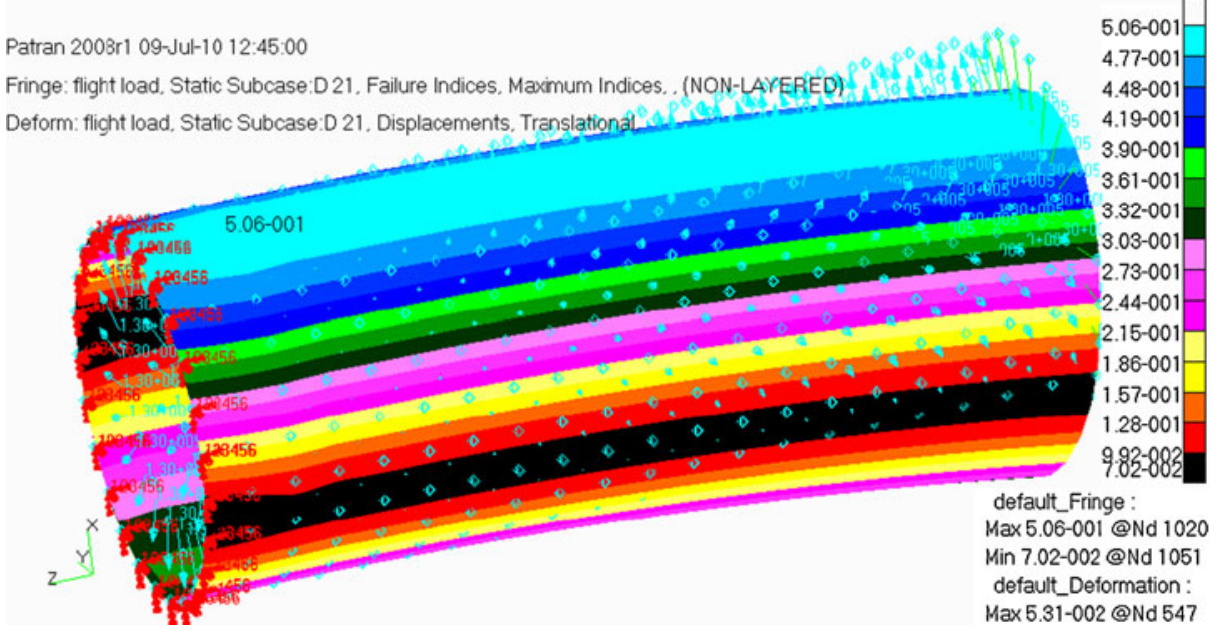

Fig. 5 The deformation and maximum failure indices after optimization

\subsection{Optimization for Sandwich Composites Cylinder with C Frame}

In Section 4.3, it can be found that too many plies are needed to achieve the requirement of the stability and the materials safety. It seems not a weight-efficient choice (the weight is $2294 \mathrm{Kg}$ ). In order to reduce the laminates thickness, C frames are added to the sandwich cylinder shell, and whether the addition of frames decreases the weight will be discussed in this section.

The initial composites layup for both skins is here $[0 / 30 / 45 / 60 / 90]_{\mathrm{s}}$. The optimization variables and their ranges are:

Frame height $H$ and width $W<20 \mathrm{~mm}, 100 \mathrm{~mm}>$

Frame thickness $t$ and $t_{1}<0.5 \mathrm{~mm}, 10 \mathrm{~mm}>$

Fiber orientation $<-90,90>$

Fig. 6 The variable history of the second step of the optimization

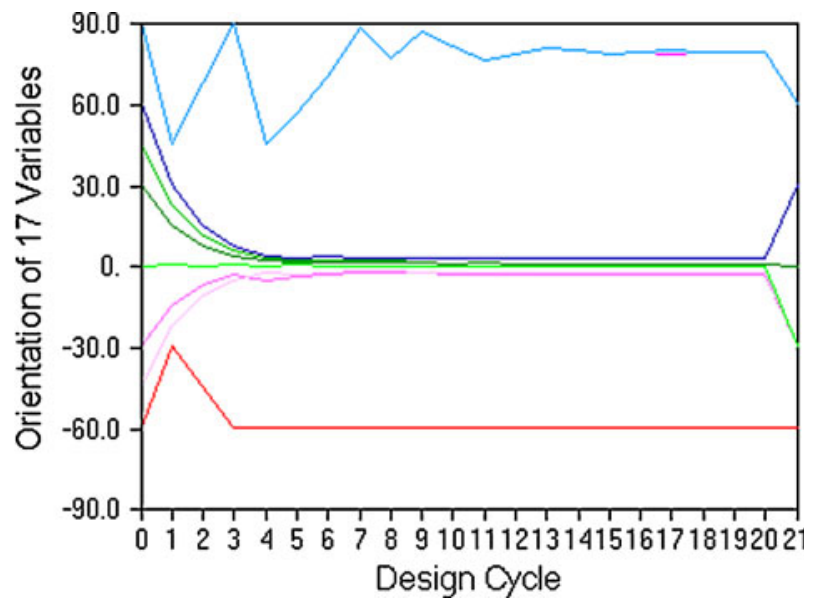




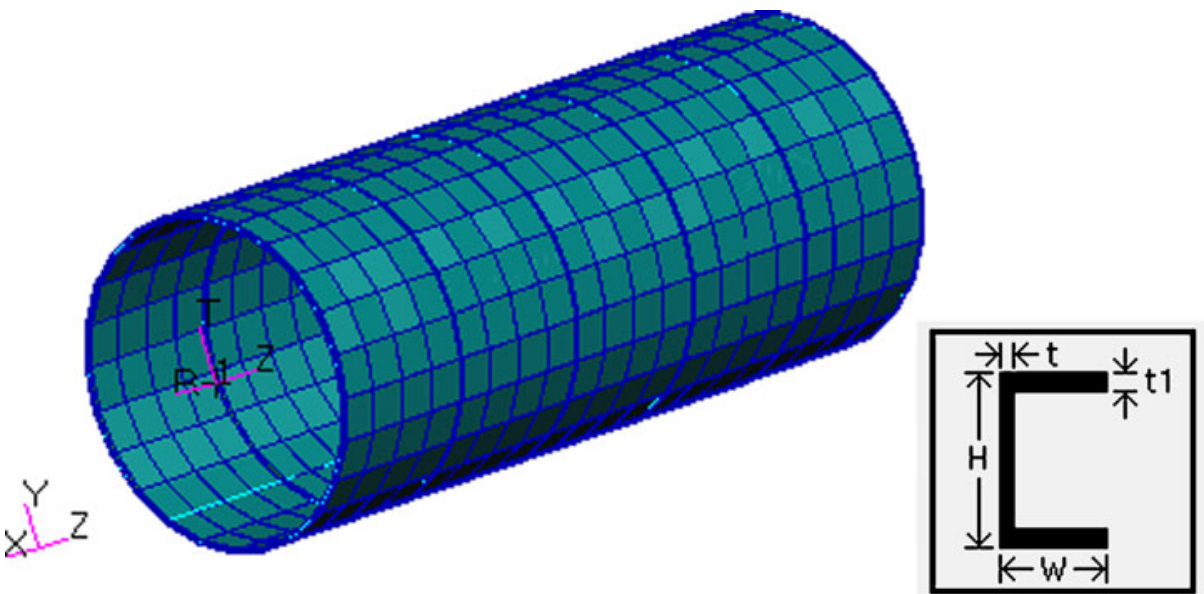

Fig. 7 Frame stiffened model and the frame sketch

Figure 8 shows the buckling eigenvalue and the total weight history. It can be seen that the eigenvalue decreases from approximately -0.35 to -1.0 , and the total weight increases from about 1150 to 1350 . Because only the frame sizes affect the total weight, the frame size history will be analyzed together with Fig. 9. From the initial design to the first design cycle, the weight decreases because the frame thickness $t$ and $t_{1}$ become lower. And the buckling eigenvalue decreases because of the frame width $W$ increases.

Figure 10 shows the deformation and the fringe of maximum failure index for composites. Because the frame spacing is $2 \mathrm{~m}$ and the cylinder length is $10 \mathrm{~m}$, there are five cyclic fringe areas along the axial direction. At the frame location, the maximum index is lower. In addition, the maximum failure index is largest at the top part of the cylinder. At this area, the maximum tensile stress exhibits. It is the same as the phenomenon in Fig. 4.

As shown in Fig. 11, the fiber orientation history is different with the trend in Fig. 6, the $0^{\circ}$ in not dominant at the final design any more. After the addition of the frame, the global buckling is probably not the critical constraint for the fiber orientation. The fiber orientation needs to be optimized to guarantee the safety of the composites. From Figs. 8 and 10, it is also found that the absolute value of the bucking eigenvalue is 1 and the maximum failure index is 0.94 (also close to 1) at the final design.

Fig. 8 The buckling eigenvalue and total weight history

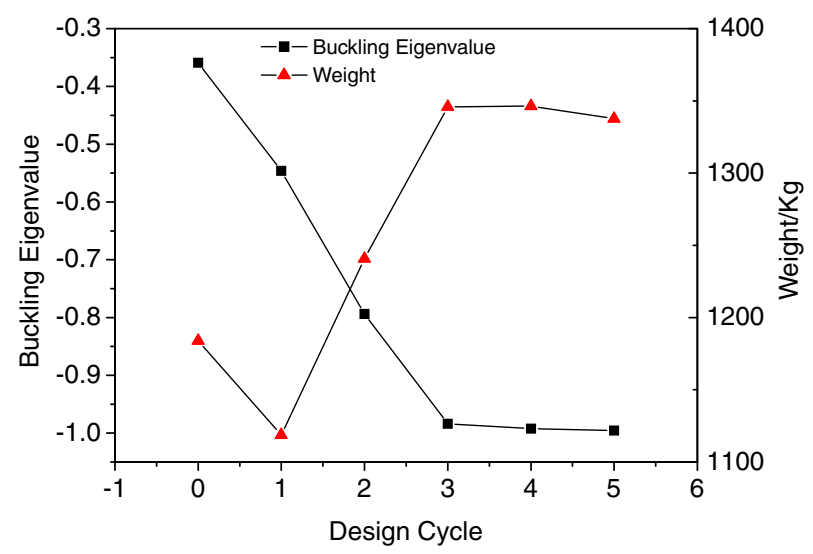


Fig. 9 The frame size history

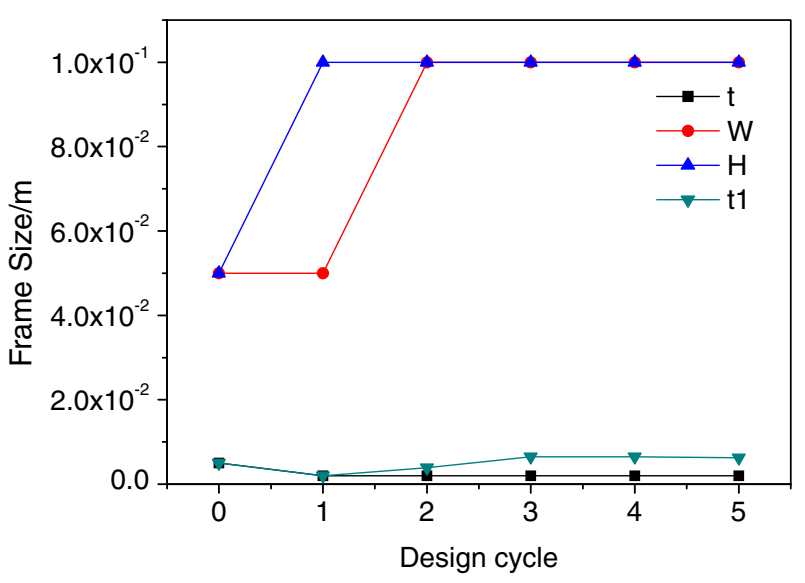

\subsection{The Core Materials}

For the sandwich structure applied in aerospace, the core materials mainly include the foam and honeycomb. They both have a relatively high transverse bending stiffness. Therefore, four kinds of honeycomb and two kinds of foam are studied for the optimum design of the sandwich composites cylinder. Their mechanical properties are listed in Appendix C. The honeycomb core is also modeled as the HEX8 element and the materials are 3D orthotropic.

After the optimization for core materials, the results are shown in Table 2. Here the maximum value of constraint denotes the extent to satisfy the constraint, when it becomes smaller the constraint requirement is better fitted. It can be seen that the sandwich structure with Rohacell 200 WF foam results in the lowest weight. And the one with Glassfiber Honeycomb core exhibits the largest weight. In addition, the fiber orientations of $30^{\circ}, 45^{\circ}$ and $60^{\circ}$ are not efficient to improve the structural stability compared with $0^{\circ}$ and $90^{\circ}$ under the load cases. Meanwhile, the frame thickness $t$ is less efficient compared to the thickness $t_{1}$ (the denotation of $t$ and $t_{1}$ can be referred to Fig. 7).

\section{Patran 2008r1 12-Jul-10 16:40:47}

Fringe: flight load, Static Subcase:D 5. Failure Indices, Maximum Indices.. (NON-LAYERED)

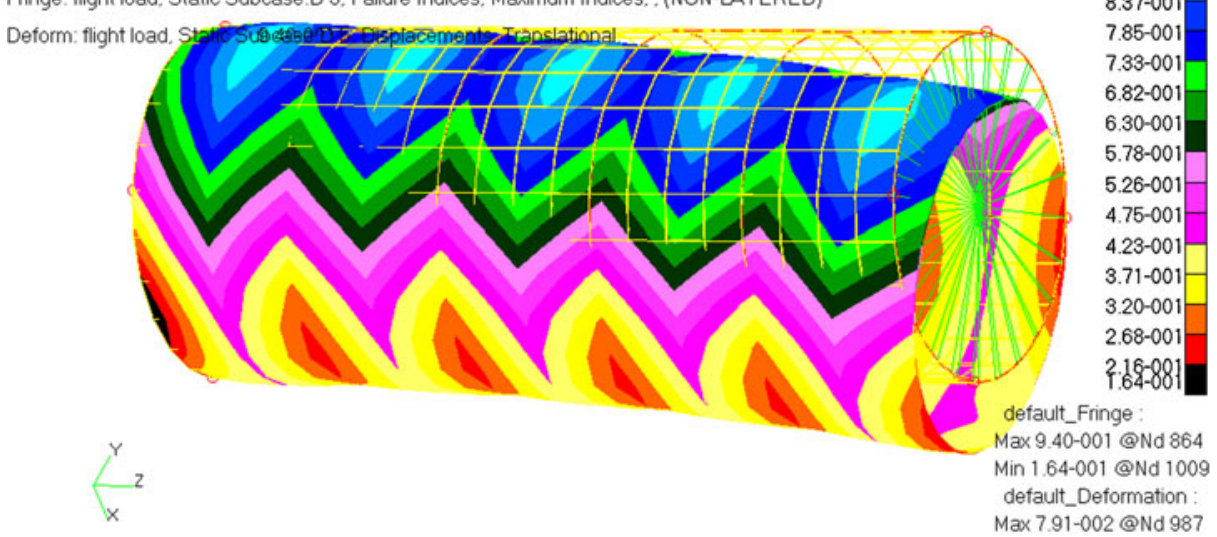

Fig. 10 The maximum failure index and deformation at the final design 
Fig. 11 The fiber orientation history

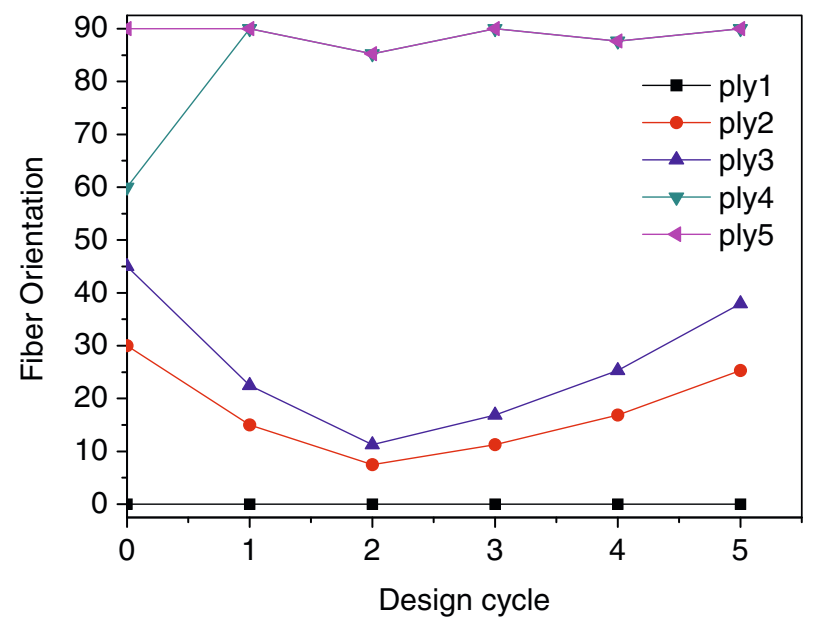

By comparison between Figs. 12 and 13, It can be found that the honeycomb core mainly endures the compressive stress under bending deformation, while the foam core mainly undergoes compressive stress at the top and bottom and tensile stress at the side part. Moreover, the stress of the honeycomb cylinder in the radial direction is even distributed while the foam core behaves largest tensile stress at the center of the frame spacing.

\subsection{ANOVA Optimization Design Towards Frame Pitch and Core}

Because the core and the frame are modeled as solid and beam elements, respectively, it is difficulty to set the frame pitch $\left(f_{\mathrm{r}}\right)$ and core thickness as variables in this model. The two variables are optimized by an orthogonal design. Each of them has three levels and the L9 orthogonal array is chosen for the analyses of the parameters on the final weight. As the Rohacell 200 WF is most efficient, it is adopted as the core materials in this section. The optimization results are shown in Table 3.

An ANOVA (Analysis Of Variance) analysis is conducted for better understanding of the influence of the two factors on the final weight. The following conclusions can be obtained based on the results of the ANOVA analysis (Table 4).

Table 2 Results of optimization for different core materials

\begin{tabular}{llllll}
\hline & Core Materials & Weight(Kg) & Max_constraint & {$[0 / 30 / 45 / 60 / 90]_{\mathrm{s}}$} & W/H/t/t1(mm) \\
\hline HONEYCOMB & Al 1/4-ACG-.003 & 1876 & 0.006 & {$[9 / 1 / 1 / 1 / 3] \mathrm{s}$} & $17 / 65 / 0.7 / 2$ \\
& $\begin{array}{l}\text { Aramid } \\
\text { HRH-10-1/8 }\end{array}$ & 1988 & 0.006 & {$[8 / 1 / 1 / 1 / 3] \mathrm{s}$} & $41 / 81 / 0.9 / 2$ \\
& Glassfiber & 2093 & 0.01 & {$[9 / 1 / 1 / 1 / 2] \mathrm{s}$} & $31 / 72 / 0.6 / 2$ \\
& HRP-3/16 & & & & \\
& Al 5/32-5052-0.002 & 1827 & 0.003 & {$[7 / 1 / 1 / 1 / 3] \mathrm{s}$} & $100 / 100 / 0.5 / 0.9$ \\
FOAM & 200 WF & 1450 & 0.02 & {$[1 / 1 / 1 / 1 / 2] \mathrm{s}$} & $100 / 100 / 0.5 / 4.8$ \\
& $110 \mathrm{WF}$ & 1833 & 0.02 & {$[8 / 1 / 1 / 1 / 3] \mathrm{s}$} & $61 / 100 / 0.5 / 1.2$ \\
\hline
\end{tabular}

Note: the term "max_constraint" denotes the extent in which a particular constraint is satisfied. A value of 0 corresponds to a reserve factor of 1 
Patran 2008r1 29-Aug-10 23:21:29

Fringe: flight load, Static Subcase:D 1_2. Stress Tensor, . X Component. (NON-LAYERED)

Deform: flight load. Static Subcase:D 1_2. Displacements. Translational.

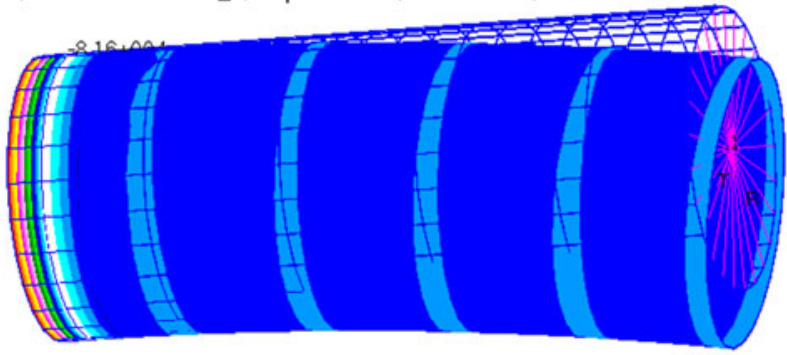

Fig. 12 The distribution of stress in radial direction at the honeycomb core

a, The core thickness gives a larger influence on the final weight than the frame pitch.

b, The final optimum weight increases with increasing the core thickness.

c, The sample with $t_{\mathrm{c}}=5 \mathrm{~mm}$ and $f_{\mathrm{r}}=0.5$ has the minimum weight.

\section{Conclusion}

In this paper, firstly an analytical criterion for face wrinkling and structural buckling under different loading cases and their combination is introduced. An optimization for sandwich cylinders is conducted based on the analytical formulae. Second, the FEM is used to

Fig. 13 The distribution of stress in radial direction at the foam core 
Table 3 Results of optimization for different frame pitch and core thickness

\begin{tabular}{llllll}
\hline Core thickness(mm) & Frame space $(\mathrm{m})$ & Weight $(\mathrm{Kg})$ & Max_constraint & {$[0 / 30 / 45 / 60 / 90] \mathrm{s}$} & W/H/t/t1(mm) \\
\hline 5 & 2 & 1419 & 0.07 & {$[4 / 1 / 1 / 1 / 2] \mathrm{s}$} & $100 / 100 / 1.9 / 4.9$ \\
& 1 & 1130 & 0.003 & {$[1 / 1 / 1 / 1 / 2] \mathrm{s}$} & $100 / 100 / 0.5 / 3.5$ \\
& 0.5 & 1129 & 0.002 & {$[1 / 1 / 1 / 1 / 2] \mathrm{s}$} & $87 / 100 / 0.5 / 2.1$ \\
10 & 2 & 1310 & 0.04 & {$[1 / 1 / 1 / 1 / 3] \mathrm{s}$} & $100 / 100 / 1.5 / 4.9$ \\
& 1 & 1249 & 0.003 & {$[1 / 1 / 1 / 1 / 2] \mathrm{s}$} & $63 / 100 / 0.6 / 5.6$ \\
& 0.5 & 1245 & 0.003 & {$[1 / 1 / 1 / 1 / 2] \mathrm{s}$} & $70 / 100 / 0.5 / 2.4$ \\
& 2 & 1450 & 0.02 & {$[1 / 1 / 1 / 1 / 2] \mathrm{s}$} & $100 / 100 / 0.5 / 4.8$ \\
& 1 & 1454 & 0.0008 & {$[1 / 1 / 1 / 1 / 2] \mathrm{s}$} & $100 / 100 / 1.7 / 2.4$ \\
& 0.5 & 1461 & 0.003 & {$[1 / 1 / 1 / 1 / 2] \mathrm{s}$} & $44 / 100 / 0.5 / 1.4$ \\
\hline
\end{tabular}

optimize the same sandwich cylinder. The FEM-based optimization results are compared with the analytical results and show a good agreement. Third, the FEM model is used to study the influence of the layer number and fiber orientation on the structural efficiency. The optimization is divided into two steps: the fiber orientation is fixed, while the optimum layer number is determined to achieve the minimum weight; then the thickness is fixed and the fiber orientations are optimized to obtain the maximum buckling load. Fourth, $\mathrm{C}$ frames are added to the sandwich cylinder to improve the structural efficiency. Lastly, different core materials are studied and an ANOVA design is introduced to determine the optimum frame space and core thickness. The following conclusions are made:

For sandwich cylinder without any stiffeners, the buckling constraint is critical. The addition of frames can efficiently improve the structural stability.

The $200 \mathrm{FW}$ foam is more efficient than honeycomb materials for the fuselage. In addition, the normal stress in the radial direction is different between the two kinds of core materials.

Table 4 The ANOVA analysis

\begin{tabular}{llll}
\hline & Core thickness & Frame pitch & Result \\
\hline Experiment 1 & 1 & 1 & 1129 \\
Experiment 2 & 1 & 2 & 1130 \\
Experiment 3 & 1 & 3 & 1419 \\
Experiment 4 & 2 & 1 & 1245 \\
Experiment 5 & 2 & 2 & 1249 \\
Experiment 6 & 2 & 3 & 1310 \\
Experiment 7 & 3 & 1 & 1461 \\
Experiment 8 & 3 & 2 & 1454 \\
Experiment 9 & 3 & 1278.3 & \\
Average of level 1 & 1226.0 & 1277.3 & \\
Average of level 1 & 1267.7 & 1393.0 & \\
Average of level 1 & 1455.0 & 115.7 & \\
Extreme Level difference & 229.0 & & \\
\hline
\end{tabular}


For the frame stiffened sandwich cylinder, the core thickness has a larger influence on the final weight than the frame spacing.

Increase of the fiber orientation in the axial direction is the most effective to increase the buckling load when the bending moment is dominant.

It is not much precise to set the layer number and orientation at the same time for the optimization using the classical algorithm. The two-step optimization is a good choice to determine the thickness and the fiber orientation.

The sandwich cylinder with core thickness of $5 \mathrm{~mm}$ and frame space of $0.5 \mathrm{~m}$ exhibit the minimum weight.

Finally, one should note that the interface between the skin and the core is not modeled in this paper. If debonding between skins and the core is one of main issues of failure modes, a bonding layer should be added into the model.

Open Access This article is distributed under the terms of the Creative Commons Attribution Noncommercial License which permits any noncommercial use, distribution, and reproduction in any medium, provided the original author(s) and source are credited.

\section{Appendix}

A. Materials and load case for cylinder sizing

$\begin{array}{lc}\text { skin laminate properties[0/45/90/-45 }]_{\mathrm{s}}(\mathrm{t}=1 \mathrm{~mm}) ; \rho_{\mathrm{f}}=1072 \mathrm{Kg} / \mathrm{m}^{3} ; \mathrm{HTA} / 977-2 \\ \text { stiffness[16] } & \text { strength[16] } \\ E_{1}=49024 & X_{\mathrm{t}}=420 \\ E_{1}=49024 & Y_{\mathrm{t}}=420 \\ G_{\mathrm{xy}}=18696 & X_{\mathrm{c}}=-407 \\ v_{\mathrm{f}}=0.31 & Y_{\mathrm{c}}=-407 \\ & S=130\end{array}$

Core properties $\left(\rho_{\mathrm{c}}=205 \mathrm{Kg} / \mathrm{m}^{3}\right)$; Rohacell 200WF foam

\begin{tabular}{|c|c|c|c|}
\hline stiffness[16] & & strength[16] & \\
\hline$E_{\mathrm{c}}=350$ & & $\sigma_{\mathrm{t}, \mathrm{c}}=5.8$ & \\
\hline$G_{\mathrm{c}}=150$ & & $\sigma_{\mathrm{c}, \mathrm{c}}=-9.0$ & \\
\hline$v_{\mathrm{c}}=0.31$ & & $\tau_{\mathrm{x}}=5.0$ & \\
\hline \multicolumn{4}{|c|}{ Flight Loadings (KNm) } \\
\hline $\mathrm{T}=900 \mathrm{KNm}$ & $\mathrm{My}=9500 \mathrm{KNm}$ & $\mathrm{Mx}=2600 \mathrm{KNm}$ & $P=0.13 \mathrm{Mpa}$ \\
\hline
\end{tabular}

B. Unidirectional Carbon ud_t300_n5208[17] for the FEM analysis in Section 4

\begin{tabular}{lllllll}
\hline$E_{1}$ & $E_{2}$ & $G_{12}$ & $G_{23}$ & $G_{13}$ & $v_{12}$ & $\rho$ \\
181000 & 10300 & 7170 & 5000 & 7170 & 0.28 & 1600 \\
$X_{\mathrm{t}}$ & $Y_{\mathrm{t}}$ & $X_{\mathrm{c}}$ & $Y_{\mathrm{c}}$ & $S$ & $F_{12}$ & \\
1500 & 40 & 1500 & 246 & 68 & 50 & \\
\hline
\end{tabular}


C. Properties of core materials

Honeycomb:

$\begin{array}{lllll}\text { Materials name } & E_{3} \text { Compression } & G_{12} & G_{23} & \rho\left[\mathrm{kg} / \mathrm{m}^{3}\right] \\ \text { Al 1/4-ACG-.003 } & 1000 & 440 & 220 & 83.3 \\ \text { Aramid HRH-10-1/8 } & 621 & 120.69 & 75.86 & 144 \\ \text { Glassfiber HRP-3/16 } & 1793 & 303 & 193 & 192 \\ \text { Al 5/32-5052-0.002 } & 2411 & 930 & 372 & 129.6\end{array}$

Foam:

\begin{tabular}{|c|c|c|c|}
\hline Materials name & $E_{1,2,3}$ & $G_{12,23,13}$ & $\rho\left[\mathrm{kg} / \mathrm{m}^{3}\right]$ \\
\hline ROHACELL $^{\circledR} 200 \mathrm{WF}$ & 350 & 150 & 205 \\
\hline ROHACELL $^{\circledR} 110 \mathrm{WF}$ & 180 & 70 & 110 \\
\hline
\end{tabular}

Notes: the unit of the modulus and strength of all materials here is $\mathrm{Mpa}$

\section{References}

1. Bitzer, T. N.: Honeycomb technology: Materials, design, manufacturing, applications and testing 1edn, 248. Springer (1997)

2. Tooren, V.: Sandwich fuselage design PhD thesis, Delft University of Technology (1998)

3. Zenkert, D.: Handbook of sandwich construction 442. Engineering Materials Advisory Services Ltd. (1997)

4. Ilcewicz, L. B. et al.: Advanced technology composite fuselage: Program Overview. Report No. NASACR-4734. Langley Research Center (1997)

5. NASA: Manual for structural stability analysis of sandwich plates and shells

6. NASA: Buckling of thin-walled circular cylinder. Report No. NASA SP-8007. NASA (1968)

7. ESDU: Buckling of thin-walled unstiffened circular cylinders under combined torsion and pressure. (1984)

8. Lennon, R.F., Das, P.K.: Torsional buckling behaviour of stiffened cylinders under combined loading. Thin Walled Struct. 38, 229-245 (2000)

9. Tennyson, R.C., Chan, K.C.: Buckling of imperfect sandwich cylinders under axial compression. Int. J. Solids Struct. 26, 1017-1036 (1990)

10. Peterson, J. \& Anderson, J.: Structural behavior and buckling strength of honeycomb sandwich cylinders subjected to bending. National aeronautics and space administration hampton va langley research center. (1965)

11. Lopatin, A.V., Morozov, E.V.: Symmetrical facing wrinkling of composite sandwich panels. J. Sandwich Struct. Mater. 10, 475-497 (2008)

12. Ley, R. P., Lin, W., Mbanefo U: Facesheet Wrinkling in Sandwich Structures. Report No. NASA/CR1999-208994. Langley Research Center (1999)

13. Stein, M., Mayers, J.: Compressive buckling of simply supported curved plates and cylinders of sandwich construction. Report No. NACA-TN-2601 (1952).

14. March, H.W.: Buckling of sandwich cylinders in torsion. Report No. FPL Report No. 1840. Forest products laboratory, Wisconsin (1958)

15. Corporation, M. S.: NX Nastran User's Guide

16. Company, D.: Development of composites technology for joints and cutouts in fuselage structure of large transport aircraft. ACEE-34-PR-3507 (1985)

17. Models, B. C.: http://www.scc.kit.edu/scc/sw/msc/Pat325/LM_Exam_1.pdf. 